\title{
The prelaparotomy diagnosis of extrahepatic biliary atresia
}

\author{
A G MANOLAKI, V F LARCHER, A P MOWAT, J J BARRETT, B PORTMANN, AND \\ E R HOWARD
}

The Department of Child Health, the Department of Nuclear Medicine, the Liver Unit, and the Department of Surgery, King's College Hospital, London

SUMMARY The diagnostic accuracy of laboratory investigations in the prelaparotomy differentiation between extrahepatic biliary atresia (EHBA) and intrahepatic disease (IHD) was assessed in 86 consecutive infants presenting with conjugated hyperbilirubinaemia. Forty five infants had EHBA and 41 IHD. The mean serum bilirubin concentration, $\gamma$-glutamyltranspeptidase (GGT) activity, and the GGT/aspartate transaminase (AST) ratio were appreciably higher in infants with EHBA than in those with IHD. In infants with IHD, however, serum bilirubin concentrations were in the EHBA range in $19(47 \%)$, as were GGT values in $29(71 \%)$, and GGT/AST ratios in $33(80 \%)$. In individual patients neither increasing nor decreasing GGT values were of diagnostic importance. Failure of biliary excretion of ${ }^{99} \mathrm{Tc}^{\mathrm{m}}$-p-Butyl-ida occurred in 29 of $30(97 \%)$ patients with EHBA but also in 22 of $23(67 \%)$ with IHD. In all 5 patients with IHD associated with $\alpha_{1}$ antitrypsin deficiency these 4 investigations gave results in the EHBA range. Liver biopsy specimen interpretation, correct in 38 of 42 infants with EHBA, gave an overall accuracy of diagnosis of $86 \%$ : the results of 3 further biopsies were equivocal. In 33 of 40 infants with IHD bile duct obstruction was excluded; the remaining 7 , including 4 with $\alpha_{1}$ antitrypsin deficiency, showed equivocal changes. Faecal excretion of ${ }^{131}$ I rose bengal faecal excretion was $<10 \%$ in 36 of 37 patients with EHBA and in 9 of 26 with IHD, giving an overall accuracy of diagnosis of $84 \%$. In patients in whom genetic disorders, such as $\alpha_{1}$ antitrypsin deficiency had been excluded, interpretation of liver biopsy specimens together with ${ }^{131}$ I rose bengal faecal excretion remain the most accurate means of identifying those who need surgery for EHBA and of avoiding unnecessary laparotomy in infants with IHD.

In untreated extrahepatic biliary atresia (EHBA) the mean age of death is 10 months and survival beyond 4 years of age is exceptional. ${ }^{1}$ Surgical developments in the past 25 years in $\mathrm{Japan}^{2}$ and elsewhere ${ }^{3}{ }^{4}$ have, however, improved the prognosis. With surgery performed before 60 days of age, bile drainage occurred in $80 \%$ of cases $^{5}$ and a 5 year survival of $34 \%$ has been achieved. ${ }^{6}$ Long term survivors are now in their third decade. ${ }^{3}$ The number of infants who benefit from surgery falls if operation is delayed ${ }^{5} \mathbf{7}$ and early surgical correction of biliary atresia is therefore essential.

Prelaparotomy diagnosis is desirable as laparotomy findings may be misleading in up to $20 \%$ of patients, and in infants with severe intrahepatic disease (IHD) and reduced bile flow the removal of narrow but patent bile ducts, incorrectly considered to be atretic, is a danger. ${ }^{9}{ }^{10}$ Also to be considered are the possible detrimental effects of laparotomy and operative cholangiography on IHD. ${ }^{11}$

Although we have found that percutaneous liver biopsy specimen interpretation and ${ }^{{ }^{131} I}$ rose bengal faecal excretion ( ${ }^{131}$ IRBE) together may discriminate between IHD and bile duct obstruction, after $\alpha_{1}$ antitrypsin deficiency has been excluded, neither investigation alone is discriminatory. ${ }^{12}$ The ${ }^{131}$ IRBE test is, furthermore, expensive in nursing time and urinary contamination of the stool may invalidate the result.

A reliable, simple, non-invasive test to differentiate between EHBA and other causes of conjugated hyperbilirubinaemia in this age group would be a major advance and $\gamma$ glutamyltranspeptidase (GGT) activity considered alone ${ }^{13}$ or expressed as a ratio of 
the aspartate aminotransferase (AST) concentration in serum ${ }^{14}$ have been reported as useful. Biliary excretion of ${ }^{99} \mathrm{Tc}^{\mathrm{m}}$-p-Butyl ida $\left({ }^{99} \mathrm{Tc}^{\mathrm{m}}\right.$ BIDA) with the appearance of the isotope in the bowel indicating a patent biliary tree has also been reported recently as a helpful investigation. ${ }^{15}$ Over a period of $3 \frac{1}{2}$ years we have compared serum GGT, GGT/AST ratio, and ${ }^{99} \mathrm{Tc}^{\mathrm{m}}$ BIDA excretion with ${ }^{131}$ IRBE and liver biopsy specimen interpretation in 86 consecutive infants investigated for conjugated hyperbilirubinaemia.

\section{Patients and methods}

EHBA was confirmed at laparotomy and by subsequent histology in 45 infants (19 boys and 26 girls). The age at investigation was mean (SD) 11.4 (6.9) weeks, range 4-34 weeks. IHD was diagnosed in 41 infants ( 28 boys and 13 girls). The age at investigation was mean (SD) $11.2(7 \cdot 2)$ weeks, range 1.5-38.5 weeks. The diagnoses included idiopathic hepatitis of infancy (29), hepatitis associated with cytomegalovirus (2), Escherichia coli septicaemia (1), $\alpha_{1}$ antitrypsin deficiency (5), and intrahepatic biliary hypoplasia (4). Six of these infants with IHD died of liver disease. No biliary tract lesion was found in the single patient who underwent necropsy. Of the 29 patients who have been followed up jaundice cleared in 26 but persists in the 3 with intrahepatic biliary hypoplasia.

GGT was measured by a Technicon SMAC high speed biochemical analyser using the modification by Vlastelica of the original method by Szasz. ${ }^{16}$ A gamma camera was used to assess ${ }^{99} \mathrm{Tcm}$ BIDA at 1,6 , and 24 hours after $5 \mathrm{mCi}$ of radionucleide was given intravenously. Complete cholestasis was excluded if isotope was identified in the gut. Excretion of ${ }^{131} \mathrm{IRBE}$ of $>10 \%$ of the intravenous dose $(1 \mu \mathrm{Ci} / \mathrm{kg})$ over a 72 hour period was interpreted as indicating that cholestasis was not complete. ${ }^{817} 18$ Percutaneous liver biopsy was performed using the Menghini technique under local anaesthesia and interpreted by 1 histopathologist (BP). ${ }^{19}$

\section{Statistical analysis}

Student's $t$ test was used for comparison of GGT and bilirubin values in EHBA and IHD and comparisons between the 2 groups of patients were by $\chi^{2}$ test.

\section{Results}

Serum GGT, GGT/AST ratio, and serum bilirubin concentrations. Serum AST, alkaline phosphatase, albumin, and prothrombin time were similar in the 2 groups of infants. Patients with EHBA had significantly higher GGT values at presentation (mean (SD), $975(518 \cdot 1) \quad \mathrm{IU} / \mathrm{l}$, range $162-2210$ IU/l) compared with infants with IHD (mean (SD), 454.1 (458) IU/1, range 29-1582 IU/1, (P<0.001)), but there was considerable overlap between the 2 groups. Twenty nine of 41 patients with IHD (70.7\%), including all 5 with $\alpha_{1}$ antitrypsin deficiency, were within the ENBA range.

Serial estimations of GGT were available in 22 infants with EHBA and 22 with IHD; preoperative values for the former group and values up to 4 weeks after presentation for the latter were considered. Eleven of 22 patients with EHBA and 12 of 22 with IHD had increasing values that exceeded $50 \%$ of the initial value in 6 infants with EHBA and 5 with IHD. Decreases in the GGT values were observed in 11 infants with EHBA and 10 with IHD but in only 2 with IHD were the decreases of $>50 \%$ of the initial value. Thus there were no consistent trends in changes in GGT values (EHBA, $\chi^{2}: 0.38$ $\mathrm{P}>0.9$ and ${ }^{99} \mathrm{Tc}^{\mathrm{m}}$ BIDA, $\left.\chi^{2}=2 \mathrm{P}>0.5\right)$.

The ratio of GGT/AST in patients with EHBA was mean (SD) $5.9(3.8)$ (range 0.5-15.9) and in patients with IHD was mean (SD) $3.9(4 \cdot 3)$ (range 0.08-16.7). Thirty eight of 45 patients with EHBA $(84.4 \%)$ had a GGT/AST ratio > 2 compared with 21 of $40(52.5 \%)$ with IHD-a significant difference $\left(\chi^{2}=10 \cdot 2 \mathrm{P}<0 \cdot 01\right)$ (Figure). Of those with IHD, $80 \%$ had ratios in the EHBA range.

Total serum bilirubin concentrations were significantly higher in EHBA patients, mean (SD) $218.6 \quad(80.8) \mu \mathrm{mol} / 1 \quad(12.78 \quad(4.72) \quad \mathrm{mg} / 100 \mathrm{ml})$, range $120-479 \mu \mathrm{mol} / 1 \quad(7.01-28.01 \mathrm{mg} / 100 \mathrm{ml})$ compared with the IHD group (mean (SD) 154.4 (129) $\mu \mathrm{mol} / 1 \quad(9.02 \quad(7.54) \mathrm{mg} / 100 \mathrm{ml})$, range $31-686 \mu \mathrm{mol} / \mathrm{l}(1 \cdot 81-40 \cdot 11 \mathrm{mg} / 100 \mathrm{ml}),(P<0 \cdot 01)$. Nineteen patients in the IHD group (47.5\%) showed values that overlapped with the EHBA range. Neither GGT/bilirubin ratios nor the product of GGT and bilirubin was helpful in distinguishing EHBA from IHD.

Isotope studies. No excretion of ${ }^{99} \mathrm{Tc}^{\mathrm{m}}$ BIDA was seen in 29 of $30(97 \%)$ patients with EHBA or in 22 of $33(66.6 \%)$ with IHD. Excretion of ${ }^{99} \mathrm{Tcm}^{\mathrm{BIDA}}$ in the IHD patients did not seem to be related to high or low serum bilirubin or GGT. Excretion of ${ }^{99} \mathrm{Tc}^{\mathrm{m}}$ BIDA did not occur in 8 of 16 infants with bilirubin values of $<120 \mu \mathrm{mol} / 1(7.01 \mathrm{mg} / 100 \mathrm{ml})$ (the lowest value in EHBA) or in 13 of 16 patients with higher concentrations $\left(\chi^{2}=3.4 \mathrm{P}>0 \cdot 1\right)$. No excretion occurred in 8 of 13 infants with GGT values of less than $162 \mathrm{IU} / 1$ (the lowest value in EHBA) or in 


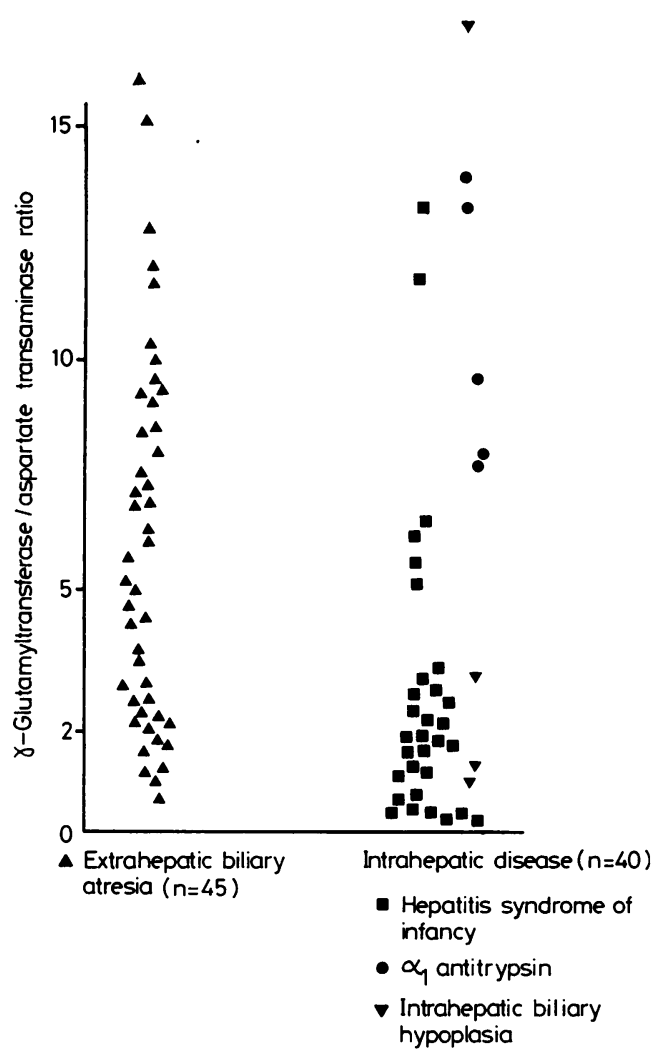

Figure $G G T / A S T$ ratio at presentation in 45 patients with extrahepatic biliary atresia and 40 patients with intrahepatic disease.

14 of 21 with higher values $\left(\chi^{2}=0.09 P>0.9\right)$. Four of 5 infants with $\alpha_{1}$-antitrypsin deficiency did not excrete ${ }^{99} \mathrm{Tcm}$ BIDA. Faecal excretion of $<10 \%$ ${ }^{131}$ IRBE occurred in 36 of 37 infants with EHBA $(97 \%)$ and in 9 of $26(35 \%)$ with IHD.

Liver biopsy specimen interpretation. A definite histological diagnosis of large duct obstruction was made in 38 of $42(90 \%)$ infants with EHBA. In 3 patients, including a 20 day old boy who had features of large duct obstruction on repeat biopsy specimen taken 20 days later, histological findings were interpreted as equivocal. One biopsy specimen showed a non-specific hepatitis. In 33 of $40(82.5 \%)$ patients with IHD the diagnosis of hepatocellular disease or intrahepatic biliary hypoplasia was made and bile duct obstruction excluded. The histological findings were equivocal in 7 patients, including 4 with $\alpha_{1}$ antitrypsin deficiency and 1 with intrahepatic biliary hypoplasia.

\section{Discussion}

Clinical features and standard liver function tests do not distinguish between infants with EHBA and those with IHD when cholestasis, evidenced by acholic stools, is complete. ${ }^{20}$ Although a recent report ${ }^{13}$ indicated that in EHBA the serum GGT values were higher than in IHD and that this was therefore a useful discriminant, the results of this study show that although the mean GGT value in EHBA was appreciably higher than in IHD, the overlap was considerable. Of IHD patients, $70.7 \%$ had values that exceeded the lowest found in EHBA. $(162 \mathrm{IU} / \mathrm{l})$. The observation that a rise in GGT values is to be expected in EHBA $^{13}$ was not confirmed in this study.

It has been suggested that a GGT/AST ratio $>2$ indicates a diagnosis of EHBA. ${ }^{14}$ This was not confirmed in 21 of 40 infants with IHD who had a ratio of $>2$, while 7 of 45 infants with EHBA had a value of $<2$. The appreciably higher serum bilirubin value found was also of little clinical relevance because of the overlap between the 2 groups.

The limitations of isotope excretion studies are well illustrated in this series. Although ${ }^{131}$ IRBE was $<10 \%$ in 36 of $37(97 \%)$ infants with EHBA, it was also equally low in 6 of $26(35 \%)$ with IHD. No isotope excretion was shown with the less time consuming ${ }^{99} \mathrm{Tc}^{\mathrm{m}}$ BIDA test in $66 \%$ of infants with IHD. It is interesting that the 1 patient with EHBA who excreted ${ }^{99} \mathrm{Tcm}$ BIDA at the same time excreted $>10 \%{ }^{131}$ IRBE. The liver biopsy specimen taken at that time indicated bile duct obstruction and when laparotomy was performed 18 days later biliary atresia was established. These observations may support the view that EHBA is a progressive disease. They also emphasise that results of isotope excretion studies may be interpreted only in the light of other investigative findings-particularly the liver biopsy specimen. ${ }^{18}{ }^{20}$ In 4 of $5 \alpha_{1}$ antitrypsin deficient patients histological features of bile duct obstruction were found, they did not excrete ${ }^{99} \mathrm{Tcm}$ BIDA, and all had GGT and GGT/AST ratios within the EHBA range.

The failure of GGT/AST and ${ }^{99} \mathrm{Tcm}$ BIDA excretion to provide an important advance in differentiation between EHBA and IHD, though disappointing in view of earlier reports, is not surprising when the many pathophysiological similarities between EHBA and IHD causes of hyperbilirubinaemia in infancy are considered. Although the use of other intrahepatic derivatives (particularly after giving phenobarbitone for 5 days) has been reported to increase the value of this investigation, ${ }^{21}$ confirmation in a larger series is required. 
We conclude that in infants in whom genetic disorders such as $\alpha_{1}$ antitrypsin deficiency or cystic fibrosis have been excluded by specific investigations, liver biopsy specimen interpretation with an overall accuracy of $86.5 \%$, together with excretion of ${ }^{131}$ IRBE that has an accuracy of $84 \%$, remain the best means of identifying infants who require surgery for EHBA and of avoiding unnecessary laparotomy in infants with intrahepatic disease.

We are grateful to Mrs Pamela Golding for typing this manuscript.

\section{References}

1 Adelmans S. Prognosis of uncorrected biliary atresia: An Update. J Ped Surg 1978; 13: 389-91.

2 Kasai MS, Suzuki S. A new operation for noncorrectable biliary atresia-hepatic portoenterostomy. Shujutsu 1959; 13: 733-9.

3 Hays DM, Kimura K. Biliary atresia: the Japanese experience. Harvard University Press, 1980.

4 Howard ER, Driver M, McClement J, Mowat AP. Results of surgery in 88 consecutive cases of extrahepatic biliary atresia. $J R$ Soc Med $1982 ; 75$ : 408-13.

${ }^{5}$ Kasai M. Results of surgery for biliary atresia. In: Javitt NB, ed. Neonatal hepatitis and biliary atresia. United States Department of Health Education \& Welfare Publication No. (NIH) 79, 1296. Bethesda, Maryland: 1979: 417-30.

${ }^{6}$ Sawaguchi S, Akiyama H, Nakajo T. Long-term followup after radical operation for biliary atresia. In: Kasai M, Shiraki K, eds. Cholestasis in infancy. Tokyo: University of Tokyo Press, 1980: 371-9.

7 Hitch DC, Shikes RH, Lilly JR. Determinants of survival after Kasai's operation for biliary atresia using actuarial analysis. J Pediatr Surg 1979; 14: 310-4.

8 Hays DM, Morton MM, Snyder WH, Reed GB, Gwinn JL, Landing BH. Diagnosis of biliary atresia: relative accuracy of percutaneous liver biopsy, open liver biopsy and operative cholangiography. J Pediatr 1967; 71 : 598-607.

9 Witzleben CL. The pathogenesis of biliary atresia. In: Javitt NB, ed. Neonatal hepatitis and biliary atresia. US Dept of Health Education \& Welfare Publication (NIH), 79-1296. Betheseda, Maryland, 1979: 339-50.
${ }^{10}$ Kahn E. Arteriohepatic dysplasia; evolution in extrahepatic biliary tree, portahepatis and hepatic parenchyma. Second International Symposium on biliary atresia. (In Press).

11 Thaler MM, Gellis SS. Studies in neonatal hepatitis and biliary atresia. Long-term prognosis of neonatal hepatitis. Am J Dis Child 1968; 116: 257-61.

12 Psacharopoulos HT, Howard ER, Portmann B, Mowat AP. Extrahepatic biliary atresia: preoperative assessment and surgical results in 47 consecutive cases. Arch Dis Child 1980; 55: 851-6.

13 Wright K, Christie DL. Use of y-glutamyl transpeptidase in the diagnosis of biliary atresia. Am J Dis Child 1981; 135: 134-6.

14 Platt MS, Potter JL, Boeckman CR, Jaberg C. Elevated GGTP/SGOT ratio. Am J Dis Child 1981 ; 135: 834-6.

15 Collier BD, Treves S, Davis MA, Heyman S, Subramanian G, McAfee JG. Simultaneous 99m Tc p-ButylIDA and I131-Rose Bengal Scintigraphy in neonatal jaundice. Radiology 1980; 134: 719-22.

16 Morgenstern S, Vlastelica B. Automated kinetic determination of gamma-glutamyl transpeptidase (abstract). Clin Chem 1976; 22 : 1224.

17 Maksoud JG, Thom AF, Kieffer J. Pinto VAC. Faecal excretion of Rose Bengal $I_{131}$ in the diagnosis of obstructive jaundice in infancy with special reference to biliary atresia. Pediatrics $1971 ;$ 48: 966-9.

18 Alvarez F, Yvart J, Odièvre M. The Rose Bengal test in neonatal cholestasis: diagnostic and prognostic value. Eur J Pediatr 1981 ; 137: 27-9.

19 Portmann B, Mowat AP, Williams R. Liver histopathology in neonates with conjugated hyperbilirubinemia. Acta Paed Belg 1976; 29 : 139-44.

20 Mowat AP, Psacharopoulos HT, Williams R. Extrahepatic biliary atresia versus neonatal hepatitis. Arch Dis Child 1976; 51 : 763-70.

21 Majd M, Reba RC, Altman RP. Hepatobiliary scintigraphy with $99 \mathrm{~m}$ Tc-PIPIDA in the evaluation of neonatal jaundice. Pediatrics $1981 ; 67$ : 140-5.

Correspondence to Dr Alex P Mowat, Department of Child Health, King's College Hospital, Denmark Hill, SE5.

Received 15 April 1983 